\title{
Aufbau der Arbeit
}

Die vorliegende Arbeit gliedert sich in vier große Bereiche: Die Darstellung des Forschungsstandes zum Untersuchungsgegenstand der Predigt innerhalb der linguistischen, soziologischen und theologischen Forschung (Teil II), der Darstellung der theoretischen Grundlagen auf dem Gebiet der Gattungsforschung (Teil III), die Übersicht über die methodischen Grundlagen sowie das Vorgehen bei Datenerhebung, Datenaufbereitung, Datenanalyse und eine Einführung in das verwendete Korpus christlicher Predigten (Teil IV) und schließlich die Darstellung der Analyseergebnisse (Teil V).

Auch wenn die Arbeit eine linguistisch-interaktional ausgerichtete Studie ist, und die bisherige Forschung auf diesem Gebiet den Schwerpunkt des Forschungsrahmens bildet (Kapitel 4 und 5), sollen beim Gang durch den Forschungsstand auch einige homiletische Annäherungen an den Gegenstand der Predigt vorgestellt werden (Kapitel 6). Im Zentrum stehen dabei vor allem Forschungslinien, die die Predigt in ihrer performativen Realisierung betrachten und empirische Zugänge gewählt haben.

Im Anschluss daran wird das für diese Arbeit grundlegende theoretische Konzept der kommunikativen Gattungen nach Luckmann vorgestellt (Kapitel 8). Darüber hinaus wird aber auch das Konzept von Bachtin zur sprachlichen Gattung thematisiert (Kapitel 7), das für die bisherige sowohl linguistische als auch theologische Forschung maßgeblicher Bezugspunkt war und das vor allem mit Blick auf die interaktiven Potenziale der Gattung auch für diese Arbeit relevant wird. In den letzten Jahren wurden diese beiden Gattungsmodelle durch Linell in seinem Konzept der kommunikativen Projekte und in Bezug auf kommunikative Praktiken diskutiert. Die vorliegende Arbeit führt diese unterschiedlichen Linien zusammen (Kapitel 9) und macht sie an der Predigt fruchtbar.

Auf dieser Grundlage werden die methodischen Forschungslinien aufgezeigt, denen die vorliegende Arbeit folgt. Zentral ist dabei die Entwicklung von 
der Ethnomethodologie (Abschnitt 10.1) hin zur Konversationsanalyse (nachfolgend auch CA; Abschnitt 10.2). In Anlehnung an die Analyseprämissen der CA orientiert sich die vorliegende Arbeit vor allem an der Erweiterung im Bereich der Multimodalität. So perspektivieren die Interaktionale Linguistik (Abschnitt 10.3) und die Multimodale Interaktionsanalyse (Abschnitt 10.4) neben den „verbalen Anteile[n]“ (Deppermann/Schmitt 2007:17) der Interaktion auch visuell-leibliche und materielle Anteile und verlagern ihr Interesse hin zur multimodalen Realisierung interaktiver Ereignisse. Damit rückt nicht mehr nur eine kommunikative Ressource in den Blick der Analyse, sondern ,das Zusammenspiel aller Ausdrucksmittel, die den Interaktionsbeteiligten zur Verfügung stehen“ (Deppermann/Schmitt 2007:17). Entsprechend dieser Ausrichtung geht es auch in der vorliegenden Arbeit um die „mikroanalytische Auswertung des in Videodokumenten eingefangenen Prozesses der multimodalen Herstellung interaktiver Ordnung“ (Deppermann/Schmitt 2007:17), d. h. um die detaillierte Betrachtung und Analyse der multimodalen Konstruktion der kommunikativen Gattung der christlichen Predigt anhand von Videodaten. Besonders in den Bereichen der Datenerhebung und der Datenauswertung müssen dann etablierte Methoden notwendig erweitert und verfeinert werden (vgl. Deppermann/Schmitt 2007:16). Aus diesem Grund stellt die vorliegende Arbeit zunächst die Entwicklungen in der Videoanalyse vor (Abschnitt 10.5) und begründet darauf aufbauend das erhobene Datenkorpus (Kapitel 11). Im Anschluss daran werden die in dieser Arbeit verwendeten Transkriptionskonventionen zur Erfassung visueller Elemente genauer vorgestellt (Kapitel 12).

Den Hauptteil der Arbeit bildet die Darstellung der Analyseergebnisse. Diese konzentrieren sich zum einen auf die institutionelle und gottesdienstliche Einbettung (Kapitel 13) sowie auf die räumliche Situierung (Kapitel 14). Zum anderen untersucht diese Studie die spezifischen Wissensbestände, die zur Realisierung der kommunikativen Gattung Predigt relevant und von den Beteiligten aktualisiert werden. Dieses Wissen zeigt sich u. a. in der multimodalen Konstitution und Konstruktion des Predigtereignisses (Kapitel 15) und dort vor allem in den Eröffnungs- und Beendigungssequenzen (Abschnitt 15.1 und Abschnitt 15.2). Darüber hinaus soll nicht nur das Wissen aufgezeigt werden, dass über die Gattung selbst vorhanden sein muss, sondern auch das Wissen, das innerhalb der Predigt relevant gesetzt wird (Kapitel 16). Im Fokus stehen neben den Wissensannahmen und den Wissenszuschreibungen, die der jeweilige Prediger gegenüber der Gemeinde vornimmt (Abschnitt 16.2), auch Formen der Wissensvermittlung (Abschnitt 16.3) und damit verbundene Verfahren der Veranschaulichung (Abschnitt 16.4). Darauf aufbauend werden Formen der Darstellung deontischer Autorität des Predigers betrachtet, die sich u. a. in der Formulierung 
von Handlungsanweisungen, moralischer Leitlinien und überzeitlicher religiöser Gewissheiten zeigen (Abschnitt 16.5 und 16.6). Ziel ist es zu bestimmen, welche Rolle Wissen und dessen Vermittlung in der Predigt spielt, um die Frage zu klären, ob man von der Predigt als einer kommunikativen Gattung der Wissensvermittlung sprechen kann (Abschnitt 16.7). Den letzten Teil der Analyse bildet die Darstellung der Dialogizität der Predigt (Kapitel 17). Dazu werden zunächst theologische und linguistische Dialogmodelle vorgestellt (Abschnitt 17.1 und 17.2), bevor unterschiedliche Beteiligungsformate der anwesenden Personen herausgearbeitet werden (Abschnitt 17.3). Der Schwerpunkt liegt hier auf der Realisierung von interrogativen Formaten mit einem unmittelbaren Sprecherwechsel sowie den interaktiven Praktiken, die dazu eingesetzt werden. Einen scheinbaren Sonderfall bildet dabei die Dialogpredigt (Abschnitt 17.4). Die Frage ist dann, ob diese neue Form der Predigt auch Auswirkungen auf die Beteiligungsformate der anwesenden Personen hat. Den Abschluss bildet die Zusammenführung der hier untersuchten dialogischen Elemente in Predigten und die Bestimmung der Dialogizität in der kommunikativen Gattung der christlichen Predigt (Abschnitt 17.5).

Open Access Dieses Kapitel wird unter der Creative Commons Namensnennung 4.0 International Lizenz (http://creativecommons.org/licenses/by/4.0/deed.de) veröffentlicht, welche die Nutzung, Vervielfältigung, Bearbeitung, Verbreitung und Wiedergabe in jeglichem Medium und Format erlaubt, sofern Sie den/die ursprünglichen Autor(en) und die Quelle ordnungsgemäß nennen, einen Link zur Creative Commons Lizenz beifügen und angeben, ob Änderungen vorgenommen wurden.

Die in diesem Kapitel enthaltenen Bilder und sonstiges Drittmaterial unterliegen ebenfalls der genannten Creative Commons Lizenz, sofern sich aus der Abbildungslegende nichts anderes ergibt. Sofern das betreffende Material nicht unter der genannten Creative Commons Lizenz steht und die betreffende Handlung nicht nach gesetzlichen Vorschriften erlaubt ist, ist für die oben aufgeführten Weiterverwendungen des Materials die Einwilligung des jeweiligen Rechteinhabers einzuholen.

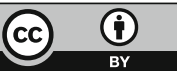

